\title{
LA SITUACIÓN DEL EMPLEO DE LOS JÓVENES Y UNA PROPUESTA DE POLÍTICAS PÚBLICAS PARA AFRONTAR EL DESEMPLEO JUVENIL*
}

\author{
THE YOUTH EMPLOYMENT SITUATION AND A PROPOSAL \\ FOR PUBLIC POLICIES TO FACE YOUTH UNEMPLOYMENT
}

\section{LA SITUATION L'EMPLOI EN LES JEUNES ET UN PROJET DE POLITIQUES PUBLIQUES POUR AFFRONTER LE CHÔMAGE JUVÉNILE}

\author{
Jordi GARCíA ViÑA*
}

\begin{abstract}
RESUMEN: La resolución de los problemas del empleo de los jóvenes requiere fortalecer los mecanismos macroeconómicos, diseñar estrategias para la formalización de la economía informal, implantar medidas de empoderamiento de las mujeres jóvenes, así como un enfoque integral que estructure políticas en educación, protección social, formación profesional y empleo.

En todo caso, cualquier política pública no sólo ha de considerar a los jóvenes de hoy, sino también es necesaria una estrategia de largo plazo que contemple factores sociales y económicos sobre trayectorias laborales de jóvenes del futuro.
\end{abstract}

Palabras clave: jóvenes, empleo, desempleo, políticas públicas.

ABSTRACT: The resolution of youth employment problems requires strengthening macroeconomic mechanisms, designing strategies for the formalization of theinformaleconomy, implementing measures for the em-

* Recibido el 27 de mayo de 2019 y aceptado para su publicación el 6 de septiembre de 2019.

** Doctor en derecho. Catedrático de derecho del trabajo y de la seguridad social en la Universidad de Barcelona.

Revista Latinoamericana de Derecho Social
Núm. 26, enero-junio de 2020, pp. 65-94 
powerment of young women, as well as a comprehensive approach that structures policies in education, social protection, training professional and employment.

In any case, any public policy must not only consider the current young people, but also a long-term strategy that contemplates social and economic factors on the work trajectories of young people in the future.

Keywords: young people, employment, unemployment, public policies.

RÉSUMÉ: La résolution des problèmes d'emploi des jeunes nécessite le renforcement des mécanismes macroéconomiques, l'élaboration de stratégies pour la formalisation de l'économie informelle, la mise en œuvre de mesures visant à autonomiser les jeunes femmes, ainsi que d'une approche globale structurant les politiques en matière d'éducation, de protection sociale et de formation. Professionnel et emploi.

En tout état de cause, toute politique publique doit non seulement prendre en compte les jeunes d'aujourd'hui, mais également une stratégie à long terme prenant en compte les facteurs sociaux et économiques sur les trajectoires de travail des jeunes du futur.

Mots-clés: jeunes, emploi, chômage, politiques publiques.

SumArio: I. ¿Cómo es el mercado de trabajo de los jóvenes? II. Algunas observaciones sobre el desempleo juvenil. III. Una propuesta sobre programas públicos en relación con el empleo de los jóvenes. IV. Bibliografía.

\section{I. ¿CÓMO ES EL MERCADO DE TRABAJO DE LOS JÓVENES?}

a realidad que se encuentran las personas jóvenes cuando acceden al mercado de trabajo presenta importantes amenazas en los próximos años, teniendo en cuenta el deterioro de las perspectivas económicas en general, y muy especialmente en los países emergentes y en desarrollo, ya que según el FMI ante un crecimiento en 2016 de un 3.1\%, que rozó un máximo de 4\% en 2017, disminuyó a 3.6\% en 2018, continúa esta trayectoria para ubicarse en $3.3 \%$ en $2019 .{ }^{1}$

1 https:// wmw.imf.org/es/Publications/WEO/Issues/2019/03/28/world-economic-outlook-april2019. 
Así, algunos países exportadores de materias primas clave de los países emergentes, entre los que se encuentran diversas naciones de Latinoamérica, presentan mayores crisis en sus economías que las previstas por los pronósticos realizados por los diferentes organismos internacionales. Además, el crecimiento en los países en desarrollo se ve obstaculizado por la volatilidad constante de los mercados de productos básicos y el debilitamiento de la demanda de los países que son socios en su actividad comercial. Finalmente, la desaceleración en los países emergentes y en desarrollo ha contribuido a atenuar los tímidos signos de recuperación en los países desarrollados, donde los pronósticos determinan un lento y escaso incremento en los próximos años, sin que bajo ningún concepto se supere el 1.5\%, según datos de la Organización Internacional del Trabajo. ${ }^{2}$

A esta situación hay que sumarle las incertidumbres que introducen la digitalización y la inteligencia artificial en la vida de los ciudadanos, en la economía y, por ende, en el mercado de trabajo, como lo muestra el Banco Mundial. ${ }^{3}$ Existe una clara inquietud de la gran mayoría de las personas en relación con los efectos que va a tener sobre las personas. En positivo, aumenta la capacidad productiva de las empresas, supone un incremento de las competencias de las personas, facilita muchos aspectos de la vida, pero también se pueden citar aspectos negativos en relación con el deterioro de las condiciones laborales, especialmente para las personas con menores conocimientos digitales.

La primera aproximación para estudiar esta materia es saber cuál es la composición de la población joven. Si se analiza desde un punto de vista histórico, hay que tener en cuenta que desde los años cincuenta del pasado siglo hasta la actualidad, según la ONU, se pueden observar tres tipos de tendencias: ${ }^{4}$

- Asia. Ha experimentado un importante incremento que está llegando a su punto álgido, a partir del cual, se observa un proceso de estancamiento con una progresiva disminución.

- África. Ha tenido un crecimiento exponencial de la década de 1990, tendencia que sigue en la misma línea en las previsiones.

- En el resto de las regiones se observan estancamientos con tendencia a la disminución.

\footnotetext{
2 OIT, World Employment Social Outlook. Trends 2019, Ginebra, pp. 5 y ss.

3 http:// documents.worldbank.org/curated/ en/636921541603308555/pdf/WDR2019-OverviewSpanish.pdf.

4 bttp:// www.un.org/en/development/desa/population/publications/pdf/popfacts/PopFacts_2015 -1.pdf.
} 
Esta revista forma parte del acervo de la Biblioteca Jurídica Virtual del Instituto de Investigaciones Jurídicas de la UNAM http://www.juridicas.unam.mx/

Esta situación se observa claramente en el siguiente gráfico. ${ }^{5}$

\author{
GRÁFICO 1
}

EVOLUCIÓN DE LA POBLACIÓN JOVEN

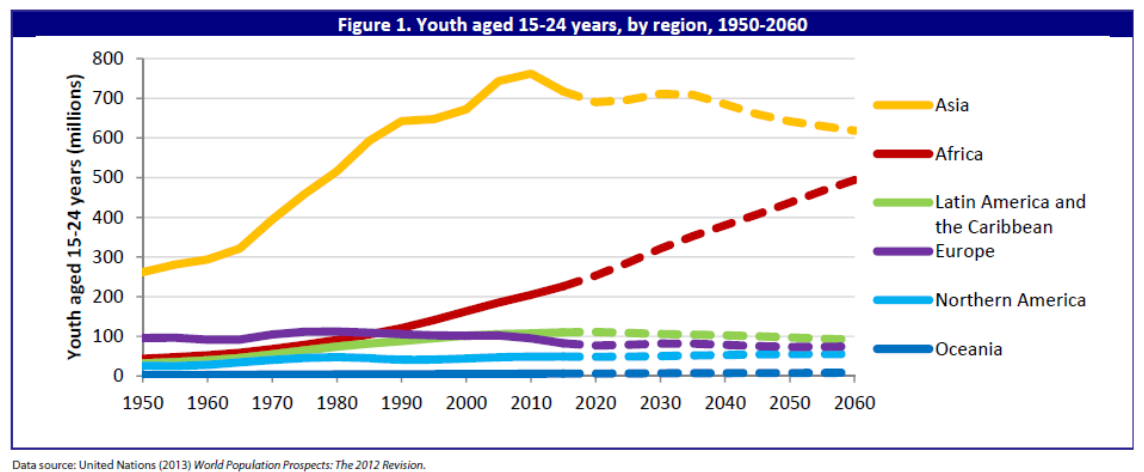

Fuente: ONU.

Con relación al futuro, y según estas proyecciones, se va a producir una disyuntiva de consecuencias en la que actualmente es difícil valorar: mientras que la población joven que representa en la actualidad el 30\% del total va a sufrir un descenso alcanzando el $20 \%$ en 2050 y el $15 \%$ en 2100 ; en cambio, la población adulta, que hoy representa menos del 10\%, se incrementará exponencialmente alcanzando el 19\% en 2050 y más del 30\% en 2100 .

Una vez determinado el porcentaje de población joven en relación con el total, hay que analizar cuáles son las principales características de los jóvenes en cuanto a su participación en el mercado laboral.

\title{
1. Informalidad laboral
}

La informalidad es uno de los principales problemas de muchos mercados de trabajo en diferentes países del mundo. Esta situación, en esta línea de afectación, impacta de manera muy relevante en las personas jóvenes tanto cuando quieren acceder a su primer puesto de trabajo, como durante su carrera profesional. Además, si estas personas presentan limitaciones en cuanto

\footnotetext{
5 ONU, Population Facts. Youth Population Trends and Sustainable Development, mayo de 2015.
} 
a su empleabilidad, por ejemplo, por falta de educación o bajos niveles de riqueza, entonces la situación se convierte en dramática. ${ }^{6}$

Los datos son muy claros en esta realidad y las diferencias radicales entre las personas jóvenes y los adultos. Así, con relación al salario, mientras los trabajadores adultos reciben una remuneración inferior al salario mínimo en un porcentaje del 10\%, el nivel de las personas jóvenes es el doble. Idéntico mismo escenario se percibe en cuanto a la duración de su contrato, el 7\% de los trabajadores adultos tienen un contrato temporal, mientras que la temporalidad se encuentra en el $23 \%$ en los jóvenes.

El empleo informal se presenta de manera diferente según las diversas categorías de trabajadores, afectando especialmente a las mujeres, jóvenes y con bajos niveles de renta (pobres).

Existe una relación directa entre la pobreza de los jóvenes y su bajo nivel educativo, especialmente porque este colectivo difícilmente accede a un puesto de trabajo que no se pueda incluir en la categoría de empleo informal.

Es evidente que existe una diáfana conexión entre los altos porcentajes de desempleo de los jóvenes, la falta de educación y la informalidad laboral. Si estas personas que normalmente no presentan ni la educación obligatoria completa logran acceder al mercado de trabajo, la posibilidad de que sólo encuentren puestos de trabajo en el sector de la economía informal y/o que los puestos de trabajo que desarrollen durante toda su carrera profesional se ubiquen en esta tipología es altísima.

\section{Escasa calidad del empleo}

Si bien es cierto que el objetivo de cualquier trabajador es tener un empleo, la evolución del mercado de trabajo, bajo la influencia de las diferentes organizaciones internacionales vinculadas con el trabajo, y especialmente la Organización Internacional de Trabajo, ha originado que se reclame algo más que la característica cuantitativa, y aparezcan elementos cualitativos, hablándose de empleos justos, decentes o de calidad.

Ahora bien, en una situación en la que las personas jóvenes tienen mayores dificultades para acceder al mercado de trabajo o, que una vez que están dentro, sus posibilidades de desarrollo de su carrera profesional son limitadas, es evidente que este colectivo de trabajadores no va a poder disfrutar de esta calidad en la actividad laboral que desarrollen.

6 Aunque no referido sólo a la informalidad de los jóvenes, véase, por su interés, bttps:// libguides.ilo.org/informal-economy-es. 
Esta peor condición se va a predicar de mayor porcentaje de contratos de duración determinada, de jornadas parciales, imposibilidad de acceder a los mecanismos de formación continua en la empresa, de imposibilidad de desarrollo de la carrera profesional, de bajos salarios o de no existencia de relación alguna con el sistema de seguridad social, por lo que no cabe ningún tipo de protección social. Así, según los últimos datos disponibles correspondientes a 2017, en los países de la OCDE, la tasa de temporalidad de los contratos de los trabajadores adultos se ubicaba en el 9.5\%, frente al 25\% de los trabajadores jóvenes y el porcentaje de parcialidad de la jornada de trabajo entre los adultos cabe determinarlo en un $11.9 \%$, mientras que las personas jóvenes estaban en el 30\%.7 Además, cuando se dan todas las circunstancias de manera conjunta, la situación de las personas trabajadoras jóvenes se agrava de manera considerable.

En esta cuestión hay que tener en cuenta la siguiente contradicción. Si bien es cierto que estas características de flexibilidad en el puesto de trabajo, preferentemente asociadas con la temporalidad y la parcialidad de la relación laboral, puede ser un mecanismo para que las personas jóvenes puedan acceder a puestos de trabajo de mayor calidad, especialmente con mayor retribución, especialmente en aquellos que presentan mayor desarrollo en su economía, hay muchos países en los que no se da esta correlación, de manera que los jóvenes que tienen este tipo de contratos de menor calidad no suelen tener acceso a otro tipo de relaciones. Esta realidad ha sido calificada por la Organización Internacional del Trabajo como de muy preocupante. ${ }^{8}$

De hecho, la mayoría de los jóvenes acepta estas condiciones porque no puede acceder a otro tipo de empleo, porque su deseo es tener derecho a un trabajo decente.

\section{La educación}

La decisión de las personas jóvenes de continuar en el sistema educativo ya sea obligatorio o superior o incorporarse al mercado de trabajo en algunos casos es simple, especialmente en países desarrollados y presenta mayor

7 https://wnw.oecd-ilibrary.org/employment/oecd-employment-outlook-2017/summary/spanish_c5c1 846a-es?parentId $=h t t p \% 3 A \% 2 \mathrm{~F} \% 2$ Finstance.metastore.ingenta.com $\% 2 \mathrm{Fcontent} \% 2 \mathrm{Fpublication} \% 2 \mathrm{Femp}$ l_outlook-2017-en.

8 https://www.ilo.org/wrmsp5/groups/public/@dgreports/@dcomm/@publ/documents/publicati on/woms_534518.pdf. 
dificultad en países en desarrollo, donde, antes de solventar esta disyuntiva, los jóvenes han de tener en cuenta diversas circunstancias especialmente económicas y sociales.

La decisión no es fácil, pues en aquellos supuestos en que tienen el deber de escoger, una de las dos opciones es claramente más ventajosa que la otra. Normalmente, cuando optan por incorporarse a un puesto de trabajo sin tener en cuenta su situación educativa se debe a necesidades económicas en general, aunque puede haber explicaciones entre los jóvenes de los países desarrollados. Por esta razón, posponer su incorporación al mercado de trabajo permaneciendo en el sistema educativo implica incrementar su empleabilidad y aumentar sus posibilidades de encontrar un trabajo de mayor valor en el momento que decidan entrar en el mercado laboral.

Evidentemente, los componentes del mercado de trabajo en relación con la situación económica pueden tener una influencia decisiva en la opción de las personas jóvenes de permanecer en el sistema educativo o ingresar en el mercado de trabajo.

Así, los jóvenes pueden decidir continuar con sus estudios, tanto si en momentos de crecimiento económico comprueban que existe un crecimiento de las oportunidades de encontrar trabajo y de calidad en posiciones en las que se necesitan personas altamente calificadas, como en situaciones de crisis económica, si existe poca oferta de empleo o los trabajos son de escasa calidad. En cambio, en aquellas situaciones económicas en las que las empresas paguen salarios altos, incluso en puestos de trabajo que no precisen de calificaciones específicas, pueden suponer la mejor excusa para los jóvenes de abandonar sus estudios y optar por estos puestos de trabajo.

Sin embargo, hay que tener en cuenta que el porcentaje de participación en la mano de obra de las personas jóvenes de edades entre 15 y 19 años es absolutamente divergente si se analizan las diferentes regiones y países en el mundo, significando esta realidad que los jóvenes encuentran diferentes pros y contras a la hora de tomar esta decisión tan trascendente.

En aquellas zonas del mundo donde la mayoría de las personas jóvenes permanecen en el sistema educativo, ya sea por motivos relacionados con la propia educación o determinadas circunstancias relativas al mercado de trabajo, el porcentaje de estas personas que tienen una relación laboral es inferior a las medias mundiales.

En cambio, en países donde la sociedad presenta situaciones económicas más devaluadas, las personas jóvenes se ven obligadas a abandonar el sistema 
educativo y admitir cualquier tipo de empleo, incluso en la economía informal, con el objetivo de poder ayudar a incrementar los ingresos en su familia.

Así, según los últimos datos que se disponen correspondientes a 2017, el porcentaje de participación de las personas jóvenes es elevado en relación con la media mundial en Asia Sudoriental y el Pacífico (32.6\%) y África Subsahariana $(45.2 \%)$. En la región de América Latina y el Caribe, aproximadamente, más de un $10 \%$ de los jóvenes combina el trabajo con los estudios, alrededor de un $21 \%$ también estudian y un $27 \%$ de los estudiantes también mientras reciben formación.

La principal consecuencia de esta realidad es que muchos jóvenes tienen un nivel de competencias bajo, especialmente en las competencias básicas, según reflejan los resultados de PISA, por lo que las empresas no encuentran la fuerza laboral que precisan. ${ }^{9}$

Una gran parte de las personas jóvenes que abandonan la escuela pasan a la inactividad o desarrollan empleos informales, teniendo en cuenta que la informalidad está más presente en el colectivo de jóvenes con situaciones de pobreza y/o vulnerabilidad. Además, las tendencias se consolidan, de manera que no es tan sencillo pasar de la economía informal a la formal, por lo que se produce una segmentación en el mercado laboral.

Hay que tener en cuenta que la mayoría de los jóvenes no sigue las etapas tradicionales de estudio, búsqueda de empleo y trabajo, sino que su situación cambia con frecuencia, por lo que los indicadores tradicionales de análisis del mercado de trabajo deben combinarse con otros mecanismos diferentes y además existe una clara brecha de género con mayor duración promedio de transición de la escuela al trabajo para las mujeres jóvenes.

\section{Jóvenes que ni estudian, ni trabajan}

Entre las personas jóvenes, es fundamental analizar cuántos de ellos pueden incluirse en el concepto de "ni-nis", cuyo grupo comprende las personas de edades entre 15 y 29 años que no tienen empleo ni cursan estudios.

\footnotetext{
9 bttp://mwn.oecd.org/pisa/pisaenespaol.htm.
} 
GRÁFICO 2

TRABAJADORES JÓVENES QUE NI ESTUDIAN NI TRABAJAN

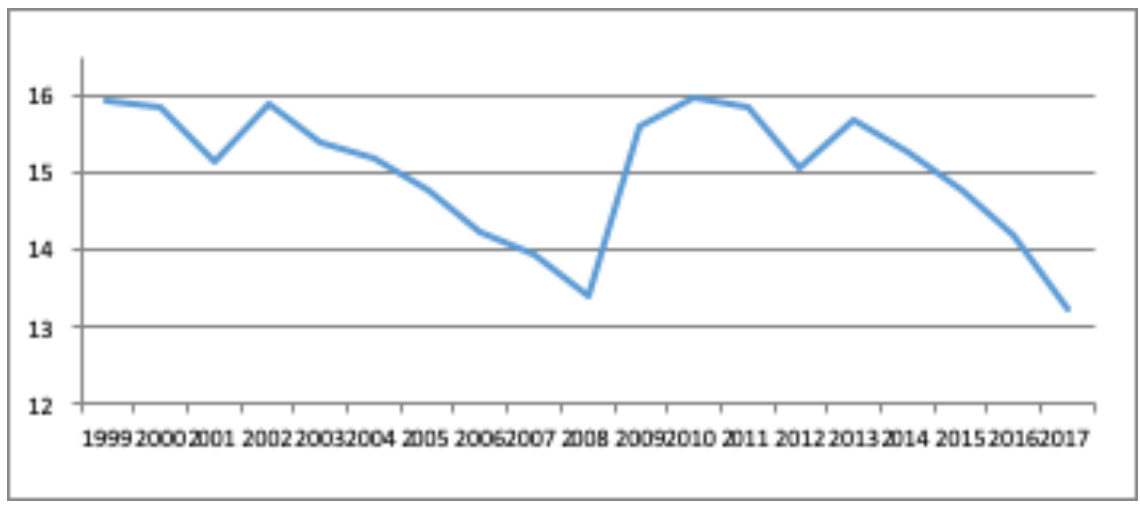

FUENTE: OCDE (2018).

En los países de la OCDE, en los que se observa una reducción en cuanto a su número en la última década, su porcentaje, según los últimos datos correspondientes a 2017 es del 13.24\%, suponiendo un 5.89\% si se escoge la franja de edad de 15 a 19 años y del $15.4 \%$ si se refiere a los jóvenes de entre 20 y 24 años. Estos datos son especialmente graves en países que presentan reducidos ingresos per cápita y relevantes porcentajes de abandono del sistema educativo, sin perjuicio que varios países de la Unión Europea presentan asimismo relevantes porcentajes de "ni-nis", como, por ejemplo, España, Grecia e Italia. ${ }^{10}$

Si se analiza separando por género, en total, los hombres representan el $10.9 \%$ y las mujeres, el $15.6 \%$. Si se separan por franjas de edad, entre 15 y 19 años, los hombres representan el 5.8\% y las mujeres el 5.9\%, mientras que la diferencia es mayor entre 20 a 24 años, ya que los hombres son el 14.1\% y las mujeres el 16.8\%. Además, entre estas personas, el 70\% de estas mujeres jóvenes realizan actividades en el sector doméstico o de cuidado de personas, sin que perciban ningún tipo de salario, mientras que los hombres jóvenes sólo representan el 10\%.

Sin embargo, la cuestión fundamental es la propia evolución, ya que debido a las características propias de la empleabilidad de este tipo de personas,

10 https:/ / www.oecd-ilibrary.org/social-issues-migration-health/ neet-youth-in-the-aftermath-of-the-crisis _5js6363503f6-en. 
radicalmente limitada, así como la dificultad de las mismas de transitar entre el sistema educativo al mercado de trabajo, el porcentaje de personas jóvenes que se incluyen en esta categoría de "ni-nis" crece exponencialmente.

Aunque pueda parecer una contradicción, el incremento del número de "ni-nis" se ha convertido en un reto especialmente importante en los países desarrollados, donde, aunque es cierto que las personas jóvenes tienen una mayor facilidad para ingresar en el sistema de educación superior, los porcentajes de este colectivo son dramáticas, no sólo entre los jóvenes que tienen entre 15 y 19 años, sino especialmente en el grupo de edad de más de 20 años, como se puede observar, por ejemplo, en los países de la Unión Europea. ${ }^{11}$

Además, es evidente que no todo el colectivo de personas jóvenes que por haberse incorporado al mercado de trabajo en su momento renunciaron al sistema educativo formal retornan a la situación retoman su formación donde la dejaron o inician otra diferente en momentos posteriores. Esta realidad presenta diferentes o tiene múltiples derivaciones tanto desde un punto de vista individual como colectivo. Las situaciones de abandono de la escolaridad, especialmente en los niveles obligatorios, tienen graves consecuencias para el joven ya que le impide un correcto desarrollo personal y limita claramente su trayectoria como trabajador, así como para la sociedad en su conjunto, tanto desde un punto de vista educativo como laboral.

De hecho, este colectivo de los jóvenes "ni-nis" que ya no tienen ningún tipo de relación con el sistema educativo, pero que tampoco presentan vínculos con el mercado de trabajo, presentan una posición totalmente externa de cualquier medida que los países puedan tener de protección social, sin que los programas de segunda oportunidad, tan comunes en los últimos tiempos, tengan una influencia positiva clara en los mismos. Por esta razón, el problema social se agrava, ya que jóvenes que podían haber tenido una incorporación a la sociedad de manera "normal", acaban convirtiéndose en un colectivo con relevante probabilidad de exclusión social.

\section{Jóvenes e inmigración}

Las altas tasas de desempleo, las relevantes opciones que presentan los trabajadores jóvenes de estar en situaciones de pobreza, así como las escasas posibilidades que tienen de encontrar empleo o de que el que tengan se sitúe

11 https:// ec.europa.eu/eurostat/statistics-explained/index.php/Statistics_on_young_people_neither_ in_employment_nor_in_education_or_training. 
en la economía informal son claras causas para que prefieran, más que permanecer en su país de origen, buscar una nueva vida en otros lugares y emigrar. Así, según los últimos datos disponibles correspondientes a 2017, según la Organización Internacional del Trabajo, cerca de 28 millones de personas migrantes tenían entre 15 y 24 años.

Sin embargo, dado el incremento de las personas que desean emigrar, principalmente pertenecientes a países emergentes y en desarrollo, este monto puede incrementarse de manera muy importante en la próxima década, aunque es cierto que la proporción de jóvenes emigrantes se ha mantenido sin grandes cambios en los últimos años. ${ }^{12}$

\section{La pobreza como mayor riesgo de los jóvenes}

La relación de los jóvenes y la pobreza es patente. Así, por ejemplo, con datos relativos a los países de Latinoamérica y el Caribe, casi el $65 \%$ de los jóvenes, que superan los cien millones, moran en hogares con altos índice de pobreza y/o vulnerabilidad, frente a las personas adultas que representan el $57 \%$. Además, la gran mayoría de estos jóvenes tienen sólo acceso a servicios sin ningún tipo de calidad y sus empleos presentan gran precariedad, por lo que sus opciones de movilidad en la sociedad en la que viven es ninguna. ${ }^{13}$

Si se analizan los datos disponibles de las últimas dos décadas, se observa que la proporción de trabajadores jóvenes pobres presenta una clara disminución progresiva, de hecho, la cuantía de estas personas que se encuentran en supuestos de pobreza moderada o extrema se va visto reducida en un 50\%.

Ahora bien, a pesar de que estos datos pueden considerarse, sin lugar a dudas, positivos, si se comparan con situaciones similares respecto a trabajadores adultos, el resultado presenta relevantes diferencias, ya que la proporción actual de trabajadores jóvenes pobres es mayor que si se trata de adultos, habiéndose ampliado las distancias entre ambos colectivos en los últimos cuarenta años, aunque el colectivo de trabajadores jóvenes pobres se ha reducido a un ritmo mayor que en el caso de los adultos. ${ }^{14}$

12 bttps:// wnw.ilo.org/global/topics/labour-migration/policy-areas/youth-and-migration/lang-es/in dex.htm.

13 CEPAL/OIT, Coyuntura laboral en América Latina y el Caribe. La transición de los jóvenes de la escuela al mercado laboral, núm. 17, octubre de 2017.

14 Sobre esta relación entre los jóvenes y la pobreza, véase https://freechild.org/youth-andpoverty/. 
En los países emergentes y en desarrollo, según los últimos datos disponibles de la Organización Internacional del Trabajo correspondientes a 2018, se considera que el $17.1 \%$ de los trabajadores jóvenes se sitúan en niveles inferiores del umbral de la pobreza extrema, mientras que la proporción de trabajadores adultos en la misma situación es del $10.9 \% .^{15}$

Son varias las explicaciones a este fenómeno y es difícil generalizar unas conclusiones que puedan servir para todos los países, ya sean desarrollados o en desarrollo, pero en todo caso, las principales razones de esta especial presencia de trabajadores jóvenes pobres, en situaciones de extrema o moderada pobreza, se deben a que se trata de personas que han abandonado el sistema educativo por necesidad, por lo que no cuentan con las competencias básicas, ni presentan ningún tipo de experiencia y porque los jóvenes presentan mayores opciones de ser contratados en puestos de trabajo en la economía informal, debido esencialmente a su baja empleabilidad.

Además, los datos se vuelven aún más complejos cuando se introducen las cuestiones de género. Así, la situación de pobreza extrema de las trabajadoras jóvenes es del $10.5 \%$, frente al $9.4 \%$ de los trabajadores jóvenes, pero estas cifras se invierten si se analizan la realidad de la pobreza moderada, donde el porcentaje de las mujeres es del 14.1\%, y el de los hombres del 17.1\%. Aunque es difícil de dar una única solución es posible que estas circunstancias se deban prioritariamente a que son muchas las trabajadoras jóvenes que prestan servicios en el servicio doméstico o tienen empleos familiares no remunerados.

\section{La presencia de las mujeres jóvenes en el mercado de trabajo}

Existen diferencias considerables entre las personas jóvenes, ya sean hombres o mujeres, si se analizan diferentes señales relativas a su presencia en el mercado de trabajo, por lo que las cuestiones de género aparecen desempeñando un papel fundamental en esta cuestión. En general, se aprecian, con independencia de los datos que se utilicen, ya sean porcentajes de participación, actividad o desempleo, una situación de total inferioridad de las mujeres jóvenes con respecto a los hombres que no sólo está presente en el mundo laboral, sino que esta situación de desventaja también dificulta su presencia en otros niveles, como, por ejemplo, económico, social y político. ${ }^{16}$

15 OIT, World Employment and Social Outlook: Trends 2019, Ginebra, 2019, p. 17.

16 https:// www.ilo.org/global/ research/global-reports/weso/trends-for-women2017/WCMS_5572 45/lang--es/index.htm. 
En primer lugar, en cuanto a la tasa de participación, según los últimos datos disponibles correspondientes a 2017, este porcentaje era del $49 \%$ referido a las mujeres jóvenes, mientras que el dígito correspondiente a los trabajadores jóvenes se situaba en el 76\%. Ahora bien, la relación entre los números de ambos colectivos, que supone una diferencia de 16.6 puntos porcentuales, no ha sufrido grandes alteraciones en los últimos 20 años, teniendo en cuenta que la proporción en el año 2000 era de 17.8 puntos porcentuales.

En segundo lugar, respecto a la tasa de actividad, es más difícil de determinar la influencia de las cuestiones de género ya que se aprecia una reducción continuada de casi 3 puntos porcentuales de esta tasa correspondiente a ambos géneros en los últimos 20 años. Sin embargo, la diferencia entre la tasa de hombres y mujeres se ha visto disminuida por causa de que la tasa de actividad entre los hombres presenta índices de disminución más acentuados que las tasas correspondientes a las mujeres.

En todo caso, las mayores diferencias con relación a la tasa de actividad entre trabajadores jóvenes mujeres y hombres se sitúan en los países emergentes correspondiendo a casi 31 puntos porcentuales, seguidas de los países desarrollados (algo más de 16 puntos porcentuales) y de los países en desarrollo (12 puntos porcentuales).

Si se concreta por regiones, las divergencias más importantes (más de 50 puntos porcentuales) se sitúan en los Estados Árabes, África del Norte y Asia Meridional, que a su vez corresponden a las tasas de actividad más bajas de las mujeres jóvenes (menos del 30\% en comparación con un porcentaje mundial del 49\%). En tercer lugar, en relación con el desempleo, es evidente que las mujeres jóvenes presentan los datos más altos de desempleo, si se compara con los trabajadores jóvenes hombres. En 2018, según datos del Banco Mundial, el porcentaje total de desempleo femenino se ubica en el $5.43 \%$ ( $4.65 \%$ si se trata de desempleo total en los hombres), cifra que presenta un continuado descenso, aunque moderado, si se compara con el 5.68\% de 2016 y el $5.52 \%$ en 2017 .

La tendencia de la separación entre ambos porcentajes para los próximos años va a ser de mantenimiento constante, en un sentido similar a lo que ha sucedido en los últimos años, por lo que va a persistir esta divergencia, aunque se espera que no aumente considerablemente. Similar realidad se constata en los años anteriores y se prevé para los futuros, aunque con unas cuantías muchos mayores, ya que los porcentajes casi son el resultado de multiplicar por tres los datos generales, cuando se refiere a desempleo de las personas jóvenes, ya sean hombres o mujeres. 
GRÁFICO 3

PorCENTAJE DE DESEMPLEO DE LOS JÓVENES POR GÉNERO

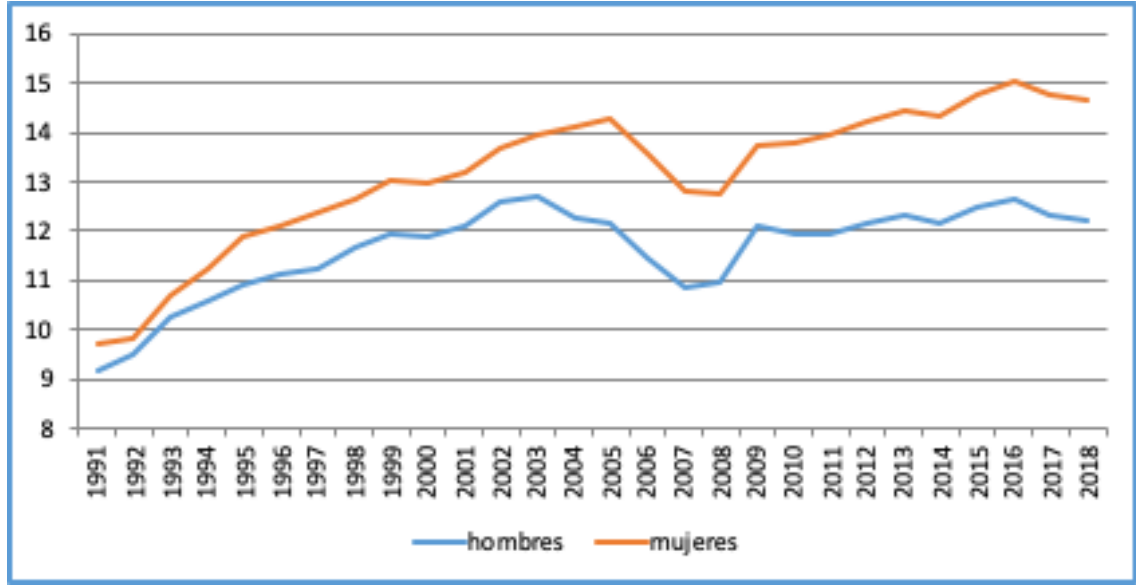

Fuente: Banco Mundial

En este caso, y como se observa en el gráfico anterior, la diferencia entre ambos porcentajes se había mantenido durante muchos años en 1 punto porcentual, siempre más elevado en las mujeres jóvenes que en los hombres, se eleva ligeramente en 2005 por encima de los dos puntos, en 2009 vuelve a reducirse a 1.6 puntos (diferencia menor de los últimos años), pero a partir de 2010 la diferencia vuelve a crecer y el dato de 2018, que corresponde a 2.43 puntos porcentuales es el mayor de toda la serie histórica. Este dato ha de hacer reflexionar a los gobernantes ya que la brecha entre géneros no sólo se consolida, sino que aumenta, se trate de momentos de desarrollo económico o de crisis, con los efectos negativos que esto supone.

Finalmente, en cuanto a las características del trabajo que desempeñan las mujeres jóvenes hay que tener en cuenta que hay un porcentaje muy importante de ellas que se encuentran en la informalidad y/o realizan actividades en el interior de familias sin recibir ninguna remuneración (tres veces más que los hombres). ${ }^{17} \mathrm{La}$ diferencia en la realización de estos trabajos familiares sin retribución, si se comparan los datos de hombres y mujeres, se ha incremen-

17 http:/ / www.youthemploymentdecade.org/en/ repor/young-women-labour-market/. 
Esta revista forma parte del acervo de la Biblioteca Jurídica Virtual del Instituto de Investigaciones Jurídicas de la UNAM http://www.juridicas.unam.mx/

tado progresivamente en los últimos años y presenta la mayor distancia en los países en desarrollo. ${ }^{18}$

\section{AlgunAS OBSERVACIONES SOBRE EL DESEMPLEO JUVENIL}

Las cifras mundiales de desempleo van a seguir creciendo en los próximos años, aunque se esperan crecimientos moderados (5.8\% en 2017 frente al $5.7 \%$ de 2016), que representan 3.4 millones de personas desempleadas más, alcanzando un total de 201 millones en 2017. Las expectativas para 2019 son de quasi estancamiento de la ratio.

Este crecimiento se debe especialmente al deterioro de los mercados laborales de los países emergentes, que van a seguir sufriendo los impactos de la crisis de 2016, pasando de un porcentaje del 5.6\% en 2016 al 5.7\% en 2017, lo que supone un incremento de 3.6 millones de desempleados. ${ }^{19}$

\section{GRÁFICO 4}

\section{EVOLUCIÓN DEL DESEMPLEO EN EL MUNDO}

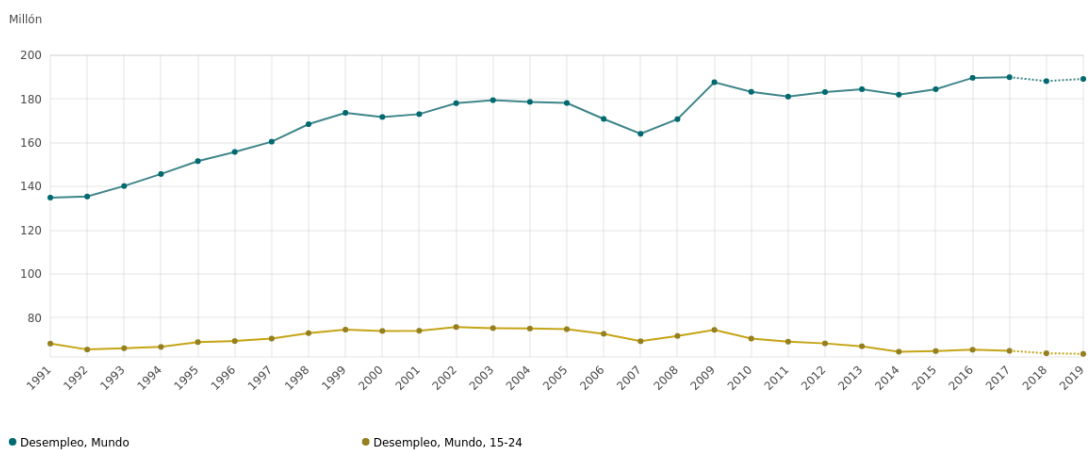

Esta dataset está armonizado y, por lo tanto, puede inferir de los datos informados a nivel nacional. Los datos para 1991-2017 son estimaciones, mientras que los del periodo 2018-2019 son proyecciones.

Fuente: Oficina Internacional del Trabajo. Estimaciones modeladas de la OIT (ilo.org/wesodata)

En cuando a los porcentajes de desempleo de las personas jóvenes, ésta se situó en el $12.21 \%$ en 2018 , viniendo del $12.69 \%$ en 2003 , cifra máxima en la serie histórica reciente.

18 bttps://www.ilo.org/wcmsp5/groups/public/---dgreports/---dcomm/---publ/documents/publication /wcms_619577.pdf.

19 bttps://www.ilo.org/wcmsp5/groups/public/---dgreports/---dcomm/---publ/documents/publication /wcms_615594.pdf. 
Estos datos suponen que en 2018 había aproximadamente 71 millones de personas jóvenes en situación de desempleo a nivel mundial, medio millón más que el año anterior. Además, se calcula que, con datos del mismo año, alrededor de 156 millones de jóvenes ocupados, es decir, el 37.7\% de los trabajadores jóvenes, vivían en una situación de extrema pobreza o moderada. ${ }^{20}$

Los datos muestran una tendencia en los últimos 25 años de índices de desempleo juvenil entre el 12 y el $14 \%$ del total del colectivo; en general, el doble de los datos para el desempleo general. Los datos parten de porcentajes del $12.1 \%$ en 1991, con un alza continuada que presenta su punto culminante en 2003 (14.3\%), con un descenso casi a datos de 1991 en 2007 (12.6\%), seguido de un crecimiento con dos picos, en 2009 (13.7\%) y 2013 (14\%).

\section{GRÁFICO 5}

\section{DESEMPLEO JUVENIL. TOTAL}

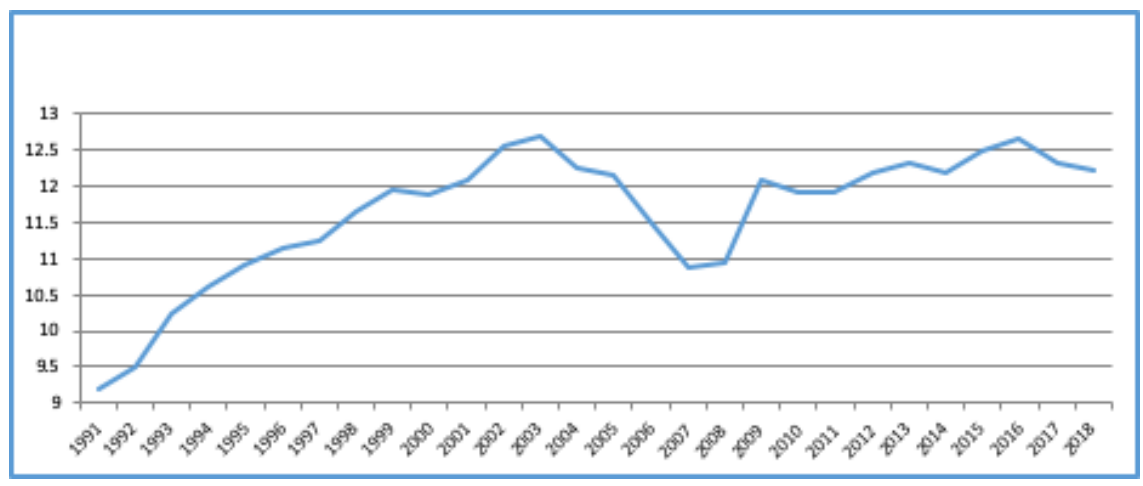

Fuente: bttps:// datos.bancomundial.org/indicator/SL.UEM.TOTL.FE.ZS.

La cuestión fundamental es determinar la evolución del desempleo juvenil para los próximos años, en los que se espera que mantenga niveles altos en términos globales, a pesar de continuar su tendencia a la baja.

Sin embargo, se aprecian relevantes diferencias en cuanto a la evolución del desempleo juvenil si se analizan agrupando los países en los dos grupos típicos de estudio: países emergentes y en desarrollo.

20 Se entiende que existe extrema pobreza cuando la persona ha de vivir con menos de 1.90 dólares por día y esta situación es calificada como moderada cuando esta renta diaria se sitúa entre 1.90 y 3.10 dólares. 
Esta revista forma parte del acervo de la Biblioteca Jurídica Virtual del Instituto de Investigaciones Jurídicas de la UNAM http://www.juridicas.unam.mx/

Concretamente, la tasa de desempleo de las personas jóvenes en los países emergentes ha pasado de un nivel de 13,3\% en 2015, hasta alcanzar el 13,6 \% en 2016 y el 13,7 \% en 2017, lo que supone un aumento de 600,000 jóvenes desempleados en comparación con 2015.

En los países en desarrollo, los datos van desde el 9.4\% en 2015, con un ascenso al $9.5 \%$ en 2016 y un retroceso hasta el $9.4 \%$ en 2017 . Ahora bien, dada la creciente cohorte de personas jóvenes que se incorporan al mercado de trabajo anualmente, el número de jóvenes desempleados en los países en desarrollo se ha incrementado en medio millón entre los años 2015 y 2017.

\section{GRÁFICO 6}

DeSEMPLEO JUVENIL (2018), POR SEXOS

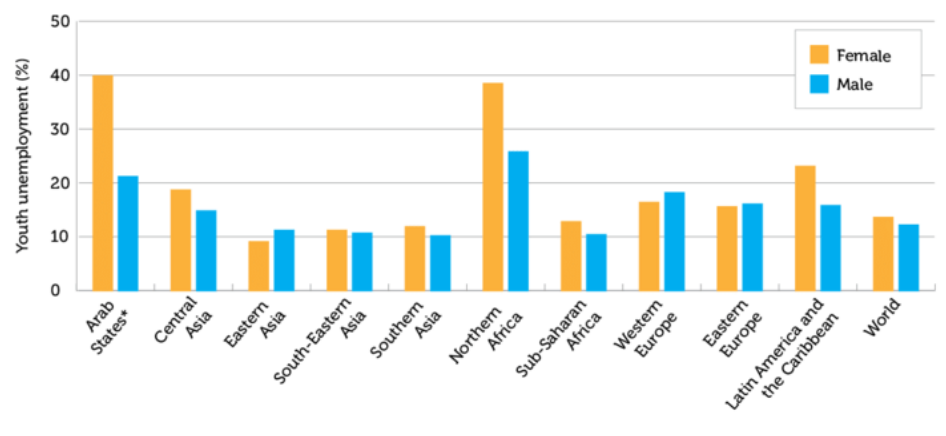

*Arab States correspond to the countries of Western Asia with the exception of Armenia Azerbaijan Cyprus, Georgia, Israel, Turkey

Source: ILO (2018b).

Fuente: ONU.

En todo caso, el hecho de que los porcentajes de desempleo de los jóvenes en los países emergentes y en desarrollo sean más bajas que las tasas correspondientes en los países desarrollados no suponen que las circunstancias del mercado laboral sean mejores en los países que se ubican en estas zonas del mundo. El significado es radicalmente diferente, ya que muestra que los jóvenes de estos países deben trabajar con mayor frecuencia en trabajos de peor calidad, que supone de manera automática una remuneración inferior, para 
poder conseguir las necesidades elementales de la vida para ellos y para sus familias, ${ }^{21}$ especialmente en relación con las mujeres jóvenes. ${ }^{22}$

Además, también hay que tener en cuenta que los periodos de desempleo de los trabajadores jóvenes son cada vez más largos, especialmente en la mayor parte de los países desarrollados. Por ejemplo, en una parte importante de los países de la OCDE, según los últimos datos correspondientes a 2017, se encontraban en desempleo por una duración superior a 12 meses un $20 \%$ del total de las personas jóvenes, mientras que en los países de la Unión Europea (UE-28), este porcentaje se situaba en el 30\%.23

Si bien es cierto que la repercusión de las situaciones de desempleo de larga duración de las personas jóvenes es menor que si se compara con el resto de grupos de edad, y este dato podría ser entendido como una diferencia positiva a favor de los jóvenes, la repercusión de esta extensa duración de la situación de desempleados tiene toda una serie de consecuencias letales para su empleabilidad ya que provocan claros deterioros en sus competencias, les impide tener la experiencia profesional que suele ser demandada en cualquier tipo de relación laboral y, progresivamente, les genera mayor desánimo en la búsqueda de trabajo, especialmente entre los colectivos de jóvenes que están buscando su primer empleo.

Esta realidad tiene varias consecuencias negativas no sólo a corto plazo, sino esencialmente a largo plazo, tanto en lo que respecta a la empleabilidad de estas personas y a su capacidad de conseguir rentas y mejorar su nivel de vida y el de su familia, así como en lo que respecta a los niveles de competitividad de las empresas y, en general, al sistema económico del país y a la sociedad en su conjunto.

En conclusión, el desafío fundamental de todos los países en relación con los jóvenes es doble, ya que, por una parte, ha de ser la reducción del desempleo juvenil por medio de la mejora del acceso a las oportunidades de trabajo y, por otro, es mejorar el trabajo que desarrollan la mayoría de las personas jóvenes que ya están trabajando, pero que se encuentran en situaciones de subempleo o en trabajos informales.

\footnotetext{
21 https:// wmw.ilo.org/womsp5/groups/public/---ed_emp/documents/publication/wcms_413826.pdf.

22 bttps:// www.un.org/development/desa/youth/wp-content/ uploads/sites/21/2018/12/WorldYo uthReport-2030 Agenda.pdf.

23 https:/ / wmw.eurofound.europa.eu/news/news-articles/the-scarring-effect-of-long-term-youth-unem ployment.
} 


\section{UNA PROPUESTA SOBRE PROGRAMAS PÚBLICOS \\ EN RELACIÓN CON EL EMPLEO DE LOS JÓVENES}

En todos los países del mundo, la situación de las personas jóvenes con relación al desempleo y/o los problemas relacionados con el desempleo de este colectivo ha estado presente en la agenda de las políticas públicas desde hace mucho tiempo, por lo que han coexistido programas y normas. Estos programas son muy diversos en aspectos tales como su concepto, metodología, desarrollo o personas implicadas y presentan características diferentes según el país que haya procedido a su implementación.

En todo caso, todas las medidas que se han llevado a cabo se pueden agrupar en cuatro grandes bloques:

- Programas de capacitación e intermediación laboral.

- Contratos de formación o de aprendizaje.

- Ayudas económicas a la contratación, en las que se incluyen rentas complementarias de salarios, exoneraciones fiscales o bonificación de cotizaciones a la seguridad social.

- Regulaciones específicas para las personas jóvenes. ${ }^{24}$

Además, por su relevancia, cabe destacar el Programa de Garantía Juvenil de la Unión Europea, único programa que tiene un ámbito supranacional y al que se están dedicando importantes cuantías de dinero. ${ }^{25}$

Los programas de políticas públicas se empezaron a implementar para tratar de dar solución a situaciones creadas por las diferentes crisis económicas, así como su consecuencia más inmediata y grave, como es el incremento del porcentaje de desempleo y suelen tener como objetivo fundamental la disminución de los niveles de pobreza a través de la prestación de trabajo.

La influencia de estas políticas en la reducción de la pobreza presenta un doble escenario: inmediato, por medio de transferencias de rentas que solventan problemas reales y concretos de estas personas y/o sus familias, y a medio y largo plazo, ya que, aumentando su empleabilidad, les permite poder acceder a puestos de trabajo con mayores remuneraciones.

${ }_{24}$ Varios autores, Políticas públicas de empleo. Un estudio desde el derecho comparado, Granada, Comares, 2013.

25 http://ec.europa.eu/social/main.jsp?catId=1079\&langId=es. Escudero Vasconez y López Mourelo, "El sistema de garantía juvenil en Europa: características, desarrollo y desafíos", Revista de Economía, núm. 881, 2014, pp. 83 y ss. 
En los últimos tiempos, las políticas activas se han especializado en reducir las desigualdades centrándose en grupos especialmente vulnerables, cuya situación depende de diversas situaciones: edad, pobreza, sexo, etcétera, creando un efecto distributivo. Es evidente, que en el marco de estas medidas, las personas jóvenes son los sustanciales protagonistas de estas políticas. ${ }^{26}$

A pesar de la implementación durante los últimos años de diversas actuaciones cuya finalidad es múltiple, ya que se refiere no sólo a conseguir que las personas jóvenes no abandonen los niveles obligatorios de educación, se consigan mayores resultados en cuanto al paso de la educación al mercado de trabajo y, fundamentalmente, tengan como consecuencia final la reducción de las tasas de desempleo juvenil, lo cierto es que los resultados de estos programas han sido muy limitados, como se puede comprobar si se analizan los datos actuales en cualquier país del desempleo de este colectivo.

Ante esta situación, aparece la necesidad de que estos programas presenten una visión exhaustiva, no sólo de corto plazo, sino también de media y larga visión y que incluya todas las medidas necesarias en campos tan diversos, pero a la vez tan imprescindibles, como son la educación, la protección social o el empleo. Estas políticas públicas han de ser implantadas con una doble finalidad muy clara, ya que han de permitir que las personas jóvenes realicen de la manera más sencilla el tránsito entre la educación y el mercado de trabajo, así como que, en aquellos casos que tengan especiales necesidades, puedan acceder a los diferentes mecanismos que se insertan en lo que se denominan servicios sociales esenciales.

Es imprescindible la conexión entre las diversas administraciones públicas de cada país a la hora de regular y ejecutar una estrategia de empleo de los jóvenes; concretamente, se deben analizar las diferentes funciones que debe llevar a cabo los diversos niveles administrativos y, teniendo en cuenta las diferentes necesidades de las personas jóvenes, requerimientos y posibilidades de cada administración, se debe proceder a realizar una evaluación cuyo objetivo fundamental sea dar el uso más eficiente y equilibrado de los recursos económicos que cada país tiene según sus presupuestos. En todo caso, cualquier política sobre empleo de los jóvenes y su desempleo no sólo ha de considerar a los jóvenes de hoy, sino también es necesaria una estrategia de largo plazo que contemple factores sociales y económicos, entre ellos de manera muy relevante la perspectiva de género, sobre trayectorias laborales de jóvenes del futuro.

26 En relación con este tipo de programas en América Latina y el Caribe, véase: https:// dds.cepal.org/bpsnc/ilp. 


\section{Cuestiones generales}

El objetivo de estos programas ha de ser revertir la destrucción de empleo que se ha producido en los últimos años, por lo que es necesario valorar las crisis económicas sufridas, así como sus repercusiones. Hay que lograr mayor dinamismo del mercado de trabajo que permita crear ofertas de trabajo. Es común que los jóvenes, especialmente los desempleados, presenten problemas de sociabilización. Por esta razón, es imprescindible incluir conceptos de sociabilización, velando especialmente porque las diferentes prestaciones y/o servicios que se ofrecen en estos programas fortalezcan el elemento de contención social. En esta línea, hay que ayudar a estos jóvenes a aumentar su autoestima, reforzar su compromiso, fomentar una actitud más activa y desarrollar el pensamiento de futuro.

Han de tener como meta reducir la informalidad, para lograr aumentar en los jóvenes la posibilidad de ser registrados, así como el desarrollo de una carrera profesional, teniendo en cuenta que, en general, el empleo no registrado no sólo se da en empresas ficticias, sino también en empresas legales. ${ }^{27}$ Es necesaria la interacción entre el sector público y el privado tanto en la configuración como en el desarrollo de estos programas, ya se incluyan en el ámbito laboral, educativo o de políticas pasivas (subsidios), así como la realización de evaluaciones a corto, medio y largo plazo de impacto teniendo en cuenta, al máximo, aspectos concretos, con el objetivo de maximizar los resultados. Finalmente, estos programas han de contar con elementos relativos a la perspectiva de género, con el objetivo claro de aumentar la presencia de las mujeres jóvenes en el mercado laboral.

\section{Los jóvenes han de ser los protagonistas de estos programas}

Los jóvenes han de ser el fundamento de este tipo de programas y todo ha de pivotar en torno a ellos, por esto, es imprescindible que el diseño esté pensado para ellos y que se conozca y se valore su grado de satisfacción. Es necesario tener en cuenta sus circunstancias, sabiendo los distintos perfiles de jóvenes que acceden a estos programas y por lo tanto las barreras a superar y las aptitudes a desarrollar.

${ }_{27}$ bttps:// staffingamericalatina.com/ en/erradicar-el-trabajo-no-declarado-para-impulsar-mercados-la borales-inclusivos/. 
Se deben analizar las barreras para el acceso de los jóvenes a estos programas, el nivel de los diferentes servicios debe dar respuesta a las necesidades que presentan los perfiles que son diferentes, hay que revisar si los procedimientos son accesibles para todos y establecer mecanismos más cercanos a los jóvenes.

\section{La finalidad de estos programas ha de ser incrementar la empleabilidad de las personas jóvenes}

Estableciendo como finalidad la mejora de la empleabilidad de los jóvenes, es fundamental marcar unos objetivos de inserción, tanto cualitativos como cuantitativos, teniendo presente elementos territoriales y temporales. A la hora de establecer estos objetivos es necesario comparar las inserciones de los jóvenes con las inserciones verificadas en una población de características similares en el mismo periodo (un grupo de control). Es estratégico analizar la tasa de éxito de todos los programas, no sólo teniendo en cuenta indicadores de actividad, sino principalmente de resultado, especialmente colocaciones y/o emprendimientos y los resultados obtenidos han de ser analizados para que permitan retroalimentar las continuas reformas normativas o de ejecución de los distintos programas. El incremento de la empleabilidad de las personas jóvenes se puede lograr si este tipo de programas combina los siguientes tres elementos. ${ }^{28}$ En primer lugar, mecanismos de introducción al trabajo. Este tipo de herramientas permite volver a poner a los jóvenes en el sistema, especialmente si fomentan opciones tales como la orientación para presentarse a una entrevista de trabajo o las recomendaciones para realizar un currículo. Es esencial en este primer nivel la presencia de las denominadas soft-skills, así como el uso de los formatos on-line en todos los programas o en una parte, dependiendo de determinados factores, por ejemplo, dificultades de acceso a los espacios físicos donde se imparten estos contenidos.

En segundo lugar, acciones de formación profesional. Es preciso que exista una gran diversidad en esta tipología de acciones, eliminando los posibles desajustes de la oferta educativa, tanto en el tiempo en relación con el inicio de los cursos, como respecto al territorio, resolviendo los problemas de coordinación, introduciendo una parte de prácticas o de formación profesional dual y programando visitas a empresas. La definición de estos cursos ha de

28 OIT, Soluciones eficaces. Politicas activas del mercado de trabajo en América Latina y el Caribe, Ginebra, OIT, 2016. 
permitir robustecer el sistema educativo y desarrollar la formación continua, armonizar la formación en los centros educativos con los programas de capacitación en el puesto de trabajo, crear programas de competencias que respondan mejor a las necesidades del mercado, establecer estrategias nacionales de mejora de las competencias e incentivar acciones de capacitación en materia de emprendimiento entre las personas jóvenes. Además, dadas las infinitas oportunidades que presenta la digitalización y las nuevas tecnologías, hay que potenciar al máximo el acceso de los jóvenes a los servicios de banda ancha, especialmente si dichos servicios se pueden ofertar de manera gratuita.

En tercer lugar, programas de emprendimiento. Es fundamental apostar por jóvenes trabajadores independientes de la economía sumergida para ayudarles en el proceso de la formalización de su actividad, ya que están más motivados para apostar por esta transformación y además el beneficio es doble, para los jóvenes y para la economía. En esta línea, es fundamental fomentar entre los jóvenes potenciales actividades que puedan desarrollar como trabajadores independientes.

En este apartado, si bien no se pueden olvidar los mecanismos que otorgan diversas modalidades de apoyo económico, los programas que presentan mejores resultados en cuanto a su consolidación posterior son los relativos a formación en dirección y administración de empresas, así las diversas modalidades de acciones de asesoramiento y orientación. Además, y aunque no se refiera exclusivamente a los jóvenes que inician una actividad empresarial, sino todas las empresas, es necesario vigorizar la relación entre estos empresarios jóvenes y las redes de empresa, instaurar mecanismos de financiación escalonados adaptados a las necesidades de los jóvenes empresarios, así como eliminar los impedimentos burocráticos a todas las empresas, facilitando al máximo la regulación para el inicio de nuevas actividades empresariales. ${ }^{29}$

\section{Necesaria conexión entre los programas de creación de empleo y el sistema educativo}

La educación formal de los jóvenes en diversas regiones del mundo es inadecuada ya que, debido a la falta de adecuación de sistemas pedagógicos, metodologías, contenidos y recursos humanos, producen un nivel bajo de

29 Miranda, Alfredo, "Políticas y leyes de primer empleo en América Latina: tensiones entre inserción y construcción de trayectorias", Revista de Ciencias Sociales, vol. 31, núm. 42, junio de 2018. 
aprendizaje y fomentan el abandono escolar, especialmente en miembros de familias de bajos recursos. Además, la ausencia de formación para el trabajo y en soft-skills y socioemocionales deja a los jóvenes desconectados con el mundo y la cultura del trabajo. Las políticas públicas que tengan como objetivo mejorar la empleabilidad de los jóvenes han de lograr que mejoren no sólo en cuanto a sus competencias técnicas, sino también respecto a las competencias básicas, que resultan ser estratégicas para dotar, especialmente a las personas jóvenes, de herramientas de flexibilidad y de adaptación al cambio que van a ser fundamentales durante toda su carrera profesional. Con esta misma finalidad, es imprescindible combinar la impartición de conocimientos teóricos, con formación de tipo práctico, ya sea relativa al puesto de trabajo o aquella que pueda otorgar capacidades para futuras ocupaciones.

En los países con problemas de bajas tasas de escolaridad o abandono relevante del sistema educativo, estos programas deben articular políticas que tengan como resultado el incremento de las ratios de escolaridad desde las edades más tempranas, disminución de las tasas de abandono, así como aumento de las competencias de lectura, escritura, comprensión y matemáticas. Así mismo, es necesario coordinar la oferta de formación profesional educativa con la laboral para que el resultado sea coherente, tenga una formulación general acercándose a conceptos de la formación profesional dual con el objetivo fundamental de incrementar la empleabilidad de los jóvenes, identificando y promoviendo nuevos campos de conocimiento, como pueden ser todos aquellos que incluyen competencias relativas a la digitalización. ${ }^{30}$

\section{Aunque existan diferentes niveles de la administración pública, uno ba de ser el protagonista}

La mayoría de los países presentan diferentes niveles en la administración pública, sin embargo, para la confección y puesta en marcha de estos programas, uno de ellos, preferiblemente el nivel superior o nacional, y con competencias en el ámbito de las relaciones laborales, ha de ser el protagonista (por ejemplo, el Ministerio de Trabajo). Este organismo ha de tener total implicación, determinando las líneas estratégicas y acciones sistemáticas, de manera que los programas sean matrices únicas de carácter nacional que permitan adaptaciones según las circunstancias. A su vez, se ha de hacer responsable

30 OIT, Trabajar para un futuro más prometedor. Comisión Mundial sobre el Futuro del Trabajo, Ginebra, OIT, 2019. 
de la optimización de las respuestas institucionales de todos los ámbitos, estableciendo mecanismos de planificación y programación en los diferentes niveles, por medio de políticas coherentes, compartiendo experiencias entre las diferentes administraciones y reforzando la coordinación entre los diferentes organismos del mismo nivel administrativo (por ejemplo, entre Ministerios) y/o ámbitos inferiores (regiones o municipios). ${ }^{31}$ Finalmente, ha de revisar procesos de circulación de la información entre los diferentes agentes, métodos de articulación, tanto formales como informales, así como necesidad de capacitación específica de los equipos técnicos de las oficinas de empleo, todo ello teniendo en cuenta la centralidad de los jóvenes en estos programas.

\section{El papel estratégico de las oficinas de empleo}

Las oficinas de empleo son estratégicas para el éxito de este tipo de programas, teniendo en cuenta la presencia de estas oficinas por todo el territorio de cada país, ya que son la gran herramienta para el conocimiento del contexto local sociolaboral. ${ }^{32}$ Es fundamental que estos programas permitan que tengan, no sólo una actuación pasiva en aplicación de las políticas del organismo responsable, sino que deben fomentar una partición activa en los mismos, de manera que no se limiten a cumplir instrucciones, sino que puedan tomar la máxima iniciativa. Estos programas han de propiciar la vinculación directa entre las oficinas de los diferentes territorios, estableciendo contactos regulares que les permitan intercambiar experiencias e información, por medio de conexión en red y/u optimización de los recursos ya existentes. Es preciso estimular su visibilidad y reconocimiento con la finalidad fundamental de definir y lograr una mejor estrategia local de comunicación, tanto con los jóvenes como con las empresas.

En muchas ocasiones, los jóvenes sólo van a las oficinas de empleo para realizar trámites formales obligatorios o cuando se les ofrece algún servicio y hay un porcentaje relevante de jóvenes que no va nunca. ${ }^{33}$ Para hacer frente a esta situación, hay que revisar las actuaciones y metas de las oficinas de

31 Varios autores, Coordinación de politicas sociales: desafios para la gestión pública, Madrid, Programa EUROsociAL, 2015.

32 bttps:// www.ilo.org/moscow/areas-of-work/employment/WCMS_384570/lang-en/index.btm. En relación con los países de la Unión Europea, véase: https://ec.europa.eu/social/main.jsp? catId $=105$ \& langId $=$ en .

33 López Mourelo, "Los servicios del mercado de trabajo en América Latina y el Caribe", Revista Vasca de Economía, núm. 93, 2018, pp. 198 y ss. 
empleo, eliminando los procesos que presentan excesiva burocracia, introduciendo mecanismos de control de los servicios, aumentando las funciones de intermediación entre oferta y demanda, mejorando los canales de comunicación con los jóvenes por medio de las nuevas tecnologías, estableciendo circuitos de seguimiento de proyectos financiados para fortalecerlos a través de diversas medidas, realizando seguimiento de las trayectorias laborales de todos los participantes en los programas e incorporando mecanismos de acompañamiento de los jóvenes como coaching o mentoring.

En cuanto a los recursos humanos de las oficinas de empleo, se han de introducir elementos de flexibilidad y actuación en función de resultados, convirtiendo a determinados servidores en "comerciales" de puestos de trabajo, centrando su activad en hablar con las empresas, ayudarlas, mantener estas relaciones. Además, es necesario reforzar la capacitación de los coordinadores de las oficinas en planificación y programación, definición de prioridades, objetivos, resultados y metas con énfasis en el seguimiento de las acciones; se debe robustecer la figura del tutor, que junto al itinerario personalizado suelen ser los elementos más valorados por los jóvenes, así como fortificar la capacitación y asistencia técnica a las personas que prestan servicios como frontline para que dispongan de los recursos técnicos profesionales para la construcción de los perfiles laborales y la orientación de los usuarios y/o beneficiarios. ${ }^{34}$

\section{Es imprescindible tener en cuenta las necesidades de las empresas y contar con su mayor implicación}

En estos programas se ha de contar con la colaboración de los otros entes implicados en el proceso, desde su diseño, implementación, ejecución y evaluación, como sindicatos, organizaciones empresariales, cámaras de comercio, organizaciones sociales, etcétera, y especialmente con las empresas privadas que son las que realmente crean ofertas y puestos de trabajo. ${ }^{35} \mathrm{Ha}$ de haber una continua relación, desde todos los puntos de vista, del organismo responsable con los empresarios, tanto desde un punto de vista general, como individual, venciendo el desconocimiento o perjuicios de los empleadores sobre estos programas. Es fundamental que todos los programas incorporen

34 Fernández Garrido, "Los retos de los servicios públicos de empleo: una visión externa”, Revista Iberoamericana de Relaciones Laborales, núm. 24, 2011, pp. 101 y ss.

35 García Solana, "La colaboración público-privada: capacidades públicas para la gestión del modelo desarrollado por el Servicio Público de Empleo Estatal y las Agencias privadas de colocación”, Cuadernos de Gobierno y Administración Pública, 4-2, 2017, pp. 135 y ss. 
mecanismos de conexión con las empresas, por ejemplo, visitas o estancias y que se puedan establecer mecanismos que incentiven el éxito de los mismos en las empresas con mejores resultados de colocación. ${ }^{36}$

Es necesario que las oficinas de empleo tengan una actitud proactiva en este proceso de relaciones con las empresas, en el que se busquen posibles experiencias de éxito y compartan buenas prácticas. ${ }^{37}$

\section{La indispensable perspectiva de género en este tipo de programas}

En la actualidad, continúan existiendo importantes diferencias en relación con el empleo de los jóvenes entre mujeres y hombres. Así, el desempleo juvenil de las mujeres es mayor, hay más mujeres que hombres desarrollando actividades en el sector informal, ya que tienen mayor propensión a ser trabajadoras familiares no remuneradas, suelen tener menor nivel educativo, sufren de manera más importante las situaciones de pobreza, existen porcentajes mayores de mujeres jóvenes "ni-nis" que hombres y, además, las mujeres que trabajan presentan claras dificultades para desarrollar su carrera profesional, por ejemplo, teniendo empleos de mayor calidad o mejor remunerados, que los hombres jóvenes. ${ }^{38}$

De la misma manera, la mayoría de las mujeres jóvenes no sigue las etapas tradicionales de estudio, búsqueda de empleo y trabajo, sino que su situación cambia con frecuencia, existiendo una clara brecha de género con mayor duración promedio de transición de la escuela al trabajo. ${ }^{39}$ Estas desigualdades en materia de oportunidades reflejan inconvenientes tanto sociales, educativos o económicos, entre otros, que suponen que estas mujeres jóvenes están en una posición peor que los hombres del mismo grupo de edad y producen diversas consecuencias, no sólo en relación con el mercado de trabajo, sino también en otros ámbitos, según la Organización Internacional del Trabajo. ${ }^{40}$ Por esta

36 Salazar-Xirinachs, "El futuro del trabajo, el empleo y las competencias en América Latina y el Caribe", Pensamiento Iberoamericano, https:// mmw.segib.org/wp-content/ uploads/07-JMS-X.pdf.

37 Banque Interaméricaine de Développement (BID), Association Mondiale des Services d'Emploi Publics (L'AMSEP) y L'Organisation de Coopération et de Développement Économiques (OECD), Le Monde des Services Publics de l'Emploi, París, 2015.

38 http:// wnw.youthemploymentdecade.org/es/repor/young-women-labour-market/.

39 Pérez del Prado, "Políticas de empleo y enfoque de género", Lan Harremanak, núm. 38, vol. II, 2017, pp. 42 y ss. btm.

40 https:// www.ilo.org/gender/Events/Campaign2008-2009/WCMS_097842/lang--en/index. 
razón, estos programas han de contener medidas de empoderamiento de las mujeres jóvenes, de manera que se fomente su participación en el mercado de trabajo, así como la regulación de políticas de conciliación y la promoción de la cultura de la corresponsabilidad en el cuidado de los hijos. Concretamente, se debe introducir la cuestión de género en todos los aspectos relativos a estos programas, eliminando cualquier tipo de barrera o brecha, formulando objetivos y acciones, analizando los resultados de los diferentes servicios según el género, así como incentivando la presencia de mujeres en aquellos que sea menor. En conclusión, cualquier tipo de medida o actuación que incremente la empleabilidad de las mujeres jóvenes. ${ }^{41}$

En todos los aspectos de estos programas, se han de tener en cuenta específicamente los aspectos de la convocatoria que van a favorecer la presencia de mujeres jóvenes, el análisis de las circunstancias que impidan su participación y mantenimiento e introducción de mecanismos concretos para remover dichos obstáculos, así como el desarrollo del papel de los diferentes servicios. ${ }^{42}$ Es fundamental en el proyecto y desarrollo de las diferentes acciones priorizar el colectivo de mujeres jóvenes, revisando el análisis de la identificación de perfiles, empezando por los de mayor vulnerabilidad, estableciendo programas diferentes según sus circunstancias personales o sociales y diseñando trayectorias claras para las mujeres jóvenes, adaptando los servicios a sus necesidades y eliminando cualquier tipo de motivo de abandono. De la misma manera, cualquier tipo de actuación debe dar respuesta a los requerimientos de los diferentes perfiles de las mujeres jóvenes, analizando si los procedimientos son accesibles para todas las usuarias y estableciendo formatos que permitan y favorezcan su asistencia a los diferentes programas. En su caso, los programas han de establecer mecanismos que eliminen las mayores dificultades de las mujeres jóvenes para combinar las actividades del hogar y de cuidado con la actividad laboral, así como las situaciones de embarazo y/o maternidad, por lo que puede ser recomendable una oferta local de servicios gratuitos y de buena calidad del cuidado, especialmente por medio de guarderías infantiles o la existencia de unas rentas que permitan el pago a terceras personas del cuidado de los hijos mientras las mujeres asisten a las actividades de los programas. Además, estos programas pueden otorgar incentivos económicos, ya sea por medio de reducción de tasas o incluso transferencia

41 bttp:// wmw.unwomen.org/es/what-we-do/economic-empowerment/facts-and-figures.

42 Díaz Muñoz, "Mujeres, trabajo y familia. Una perspectiva de género desde América Latina”, Géneros, Multidisciplinary Journal of Gender Studies, vol. 6, núm. 3, octubre de 2017, pp. 1439 y ss. 
de rentas, a las mujeres jóvenes que estudien cualquier tipo de formación que incluya las denominadas competencias STEM (Ciencias, tecnologías, ingenierías y matemáticas) o en programas de formación profesional de alto valor estratégico para la economía. ${ }^{43}$ Finalmente, se debe implantar programas de mejora del emprendimiento de las mujeres jóvenes, por medio de un apoyo financiero extraordinario, especialmente cuando les pueda faltar experiencia o garantías, así como incrementar este tipo de medidas de mejora de la empleabilidad en aquellas actividades, sectores o lugares en los que se considere que las mujeres están subrepresentadas. ${ }^{44}$

\section{BIBLIOGRAFÍA}

Banco Interamericano de Desarrollo, Programa de capacitación para jóvenes, 2017, disponible en: http:// wmw.iadb.org/es/proyectos/project-information-page,1303.ht ml?id=tc9505465.

CEPAL/OIT, Coyuntura laboral en América Latina y el Caribe. La transición de los jóvenes de la escuela al mercado laboral, núm. 17, octubre 2017.

DEMA, G. et al., ¿Qué sabemos sobre los programas y politicas de primer empleo en América Latina?, disponible en: http://www.ilo.org/wcmsp5/groups/public/--americas/---ro-lima/documents/publication/woms_369021.pdf.

DíAz MuÑoz, J. G., "Mujeres, trabajo y familia. Una perspectiva de género desde América Latina", Géneros, Multidisciplinary Journal of Gender Studies, vol. 6, núm. 3, octubre de 2017.

Escudero Vasconez, V. y López Mourelo, E., "El sistema de garantía juvenil en Europa: características, desarrollo y desafíos", Revista de Economía, núm. 881, 2014.

FERNÁNDEZ GARRIDO, J., "Los retos de los servicios públicos de empleo: una visión externa", Revista Iberoamericana de Relaciones Laborales, núm. 24, 2011.

García SolanA, M. J., “La colaboración público-privada: capacidades públicas para la gestión del modelo desarrollado por el Servicio Público de Em-

43 https:// unesdoc.unesco.org/ark:/48223/pf0000366649.

44 Sánchez-Castañeda, "Los jóvenes frente al empleo y el desempleo: la necesaria construcción de soluciones multidimensionales y multifactoriales", Revista Latinoamericana de Derecho Social, núm. 19, 2014, pp. 133 y ss. 
pleo Estatal y las Agencias privadas de colocación", Cuadernos de Gobierno y Administración Pública, 4-2, 2017.

ILO, World Employment Social Outlook, Trends for Youth, Geneva, 2016.

ILO, World Employment Social Outlook. Trends 2019, Ginebra.

ILO, A Snapshot of the Joint EC-ILO Action on Youth Employment Policies-Youth Guarantee Component, marzo 2017.

ILO, Soluciones eficaces. Politicas activas del mercado de trabajo en América Latina y el Caribe, Ginebra, OIT, 2016.

ILO, World Employment and Social Outlook: Trends 2019, Ginebra, 2019.

ILO, Trabajar para un futuro más prometedor. Comisión Mundial sobre el Futuro del Trabajo, Ginebra, OIT, 2019.

ILO, World Employment Social Outlook. Trends for women. 2017.

López Mourelo, E., "Los servicios del mercado de trabajo en América Latina y el Caribe", Revista Vasca de Economía, núm. 93, 2018.

Miranda, A. y Alfredo, M., "Políticas y leyes de primer empleo en América Latina: tensiones entre inserción y construcción de trayectorias", Revista de Ciencias Sociales, vol. 31, núm. 42, junio de 2018.

OCDE, Employment Outlook, 2018.

ONU, Youth Civic Engagement, Nueva York, 2016.

Population Facts. Youth Population Trends and Sustainable Development, mayo de 2015.

PÉreZ Del Prado, D., "Políticas de empleo y enfoque de género", Lan Harremanak, núm. 38, vol. II, 2017.

SÁNCHEZ-CASTAÑEDA, A., "Los jóvenes frente al empleo y el desempleo: la necesaria construcción de soluciones multidimensionales y multifactoriales", Revista Latinoamericana de Derecho Social, núm. 19, 2014.

Varios autores, Politicas públicas de empleo. Un estudio desde el derecho comparado, Granada, Comares, 2013.

Varios autores, Coordinación de politicas sociales: desafios para la gestión pública, Madrid, Programa EUROsociAL, 2015.

World Economic Forum, The Global Competitiveness Report. 2017-2018, Ginebra, 2017. 